\title{
Prevalencia de traumatismos dentoalveolares en pacientes infantiles del complejo asistencial Dr. Sótero del Río
}

\section{Prevalence of dental trauma of infants attended at Dr. Sótero del Río Hospital}

\author{
Castro Brezzo PF${ }^{1}$, Dreyer Arroyo $E^{2}$
}

\begin{abstract}
RESUMEN
En el presente estudio descriptivo de incidencia, 604 niños menores de 15 años fueron examinados y atendidos en la Clínica de Odontopediatría del Complejo Asistencial Dr. Sotero del Río, con diagnóstico de Traumatismo Dentoalveolar (TDA), desde Enero a Diciembre del 2010. El propósito de este estudio fue caracterizar los TDA de acuerdo a la clasificación de Andreasen y Andreasen, determinar su frecuencia y distribución estacional, tipo y número de dientes afectados, distribución de género y edad, lugar de ocurrencia y causa. Para ambas denticiones, en conjunto, más de dos dientes comprometidos presentaron una frecuencia de $58.77 \%$. Los análisis se dividieron por dentición y hubo 636 dientes definitivos y 414 temporales. En dentición definitiva se observó una mayor frecuencia en Mayo (15.36\%), Abril (13.02\%) y Noviembre (13.02\%). Las fracturas coronarias no complicadas son las más frecuentes $(40.88 \%)$ y los dientes más afectados fueron los incisivos centrales maxilares $(77.66 \%)$. Los niños sufren más TDA que las niñas (64.97\%). La mayor frecuencia de TDA para ambos géneros es a los 8 años. La principal causa fue caídas en el colegio $(46.87 \%)$. En dentición temporal se observó mayor cantidad de casos en primavera-verano $(53.74 \%)$. Siendo más frecuente en los meses Noviembre y Febrero (30.84\%). Subluxación fue el diagnostico más frecuentes con un $39.61 \%$. Los dientes más afectados fueron los incisivos centrales maxilares en un $82.13 \%$. El género masculino fue el más afectado $(62.56 \%)$. La edad en que se observó mayor frecuencia de TDA fueron los 2 años. La principal causa fue caídas en la casa (29.07\%).
\end{abstract}

Rev. Clin. Periodoncia Implantol. Rehabil. Oral Vol. 5(3); 128-131, 2012.

Palabras clave: Traumatismo dental, fracturas dentarias, luxaciones dentarias.

\begin{abstract}
In this incidence descriptive study 604 infants less than fifteen years of age were examined and attended at the Pedodontic Clinic from Dr. Sótero del Río hospital with a diagnostic of dental trauma between January and December 2010. The purpose of this research protocol was to characterize dental trauma according to the Andreasen and Andreasen classification, and also to determine their frequency, seasonal distribution, type and number of the affected teeth, place of occurrence and trauma's cause. All diagnostics were divided by dentition, 636 were from permanent and 414 from deciduous dentition. For both dentitions trauma in two teeth and more were in a frequency of $58.77 \%$. On permanent dentition a mayor frequency of trauma was observed in May (15.36\%), April (13.02\%) and November (13.02\%). The most frequent trauma was uncomplicated crown fracture (40.88\%) and the most frequent teeth affected were the upper central incisors $(77.66 \%)$. Boys suffered more trauma than girls $(64.97 \%)$. The highest frequencies of trauma for both genders occurred at eight years of age. The main causes of traumas were: falls at school (46.87\%). On deciduous dentition a mayor frequency of trauma was observed in spring and summer $(53.74 \%)$ where November and February concentrated the highest rates (30.84\%). Subluxation was the most frequent diagnostic at $39.61 \%$. The most frequent teeth affected were also the upper central incisors at $82.13 \%$. Boys also suffered more trauma than girls $(62.56 \%)$. The highest frequencies of trauma for both genders occurred at two years of age. The main cause was home falls $(29.07 \%)$.
\end{abstract}

Rev. Clin. Periodoncia Implantol. Rehabil. Oral Vol. 5(3); 128-131, 2012.

Key words: Dental trauma, tooth fractures, dental luxations.

\section{INTRODUCCIÓN}

Estudios internacionales revelan que los Traumatismos Dentoalveolares (TDA) son lesiones frecuentes en la población infantojuvenil y la literatura reporta que un $25 \%$ de todos los estudiantes han experimentado algún tipo de traumatismo ${ }^{(1,2,3)}$, cuyas causas son cada vez más diversas, y han tenido un aumento alarmante en los últimos tiempos; además pueden dejar graves secuelas, tanto funcionales como estéticas y/o sicológicas que podrían implicar tratamientos para el resto de su vida ${ }^{(2,3,4)}$. En Chile existen pocos estudios de TDA ${ }^{(5-10)}$, uno realizado por Soto L, 2007, demostró que la prevalencia de TDA a nivel nacional, en la población de 12 años, era de $5 \%$ y en la Región Metropolitana de Santiago la tasa era de $2.14 \%^{(10)}$. Debido a su frecuencia se han convertido en un problema de salud pública, por lo cual han sido incluidos en el tratamiento de urgencia odontológica en el tercer régimen de las
Garantías Explicitas en Salud (GES-AUGE) 2007-2008(11).

Además, la Salud Bucal es un tema que continuamente ha sido priorizado por la población y las autoridades de gobierno, posiblemente por los cambios socioeconómicos y culturales que han ido ocurriendo en el país, de hecho fueron incluidas en los Objetivos Sanitarios de la década 2000-2010.

La población menor de 15 años de la región metropolitana de Santiago de Chile se estima en 1.507 .655 habitantes, de ellos un $23.4 \%$ (352.137 hbts.) reciben atención en el Servicio de Salud Metropolitano Sur Oriente (SSMSO), cuyo único centro de derivación Odontopediátrico, se encuentra en el Complejo Asistencial Dr. Sótero del Río(12,13).

La muestra examinada correspondió al total de pacientes menores de 15 años (604 niños) atendidos con diagnóstico de TDA en la Clínica de Odontopediatría del Complejo Asistencial Dr. Sótero del Río (CASR) durante el año 2010.

1. Odontopediatra. Complejo Asistencial Dr. Sótero del Río. Chile.

2. Especialista en Rehabilitación Oral. Departamento de Odontología Conservadora, Facultad de Odontología, Universidad de Chile. Chile.

Correspondencia autor: Paola Castro Brezzo. paolafcastro@gmail.com. Servicio de Estomatología, Complejo Asistencial Dr. Sótero del Río. Santiago, Chile. Trabajo recibido el 23/04/2012. Aprobado para su publicación el 29/10/2012. 
El propósito de este estudio, descriptivo de incidencia, fue caracterizar los TDA de acuerdo a la clasificación Andreasen y Andreasen, determinar la frecuencia de ellos en dentición temporal y permanente según mes con mayor frecuencia, tipo y número de dientes afectados, género y edad, asociación con accidentes escolares y causa. De modo de conocer la realidad de los TDA en el SSMSO, información que puede servir para reforzar campañas preventivas de mayor impacto en la población infantojuvenil asociadas al lugar de ocurrencia más frecuente de los TDA para optimizar los recursos existentes y generar elementos adicionales en la toma de decisiones; también estimular las inversiones para la investigación tal como es una de las propuestas de las Metas 2011-2020(14). Además, promover la capacitación de los funcionarios que atienden estos pacientes ya que los centros de atención de urgencia odontológica, son puntos críticos y por lo tanto deben estar equipados para diagnosticar, brindar el tratamiento básico más adecuado para resolver la urgencia y asegurar una apropiada derivación de los pacientes hacia los otros niveles de atención cuando corresponda ${ }^{(15)}$.

\section{METODOLOGÍA}

Se realizó un estudio descriptivo de incidencia desde el 1 de Enero al 31 de Diciembre del 2010, en que tres operadores se calibraron previamente, examinando 10 pacientes que habían sufrido TDA con anterioridad al inicio del trabajo, obteniendo un resultado de kappa 0,79.

Se examinaron la totalidad de los pacientes entre 0 y 14 años 11 meses derivados a la Clínica de Odontopediatría, desde el Servicio de Urgencia del Complejo Asistencial Dr. Sótero del Río con diagnóstico de TDA.

A todos los niños se les realizó un examen clínico (visual y táctil) y radiográfico para catalogar los TDA de acuerdo a la clasificación Andreasen y Andreasen ${ }^{(16)}$. Los datos obtenidos fueron consignados en una hoja para completar, diseñada para tal efecto, consignándose en las columnas las variables a estudiar y en las filas los niños, no se registraron los nombres de los pacientes solo se asignó número, para evitar discriminación arbitraria de estos y no se incluyeron variables socioculturales solo aquellas que se deseaban estudiar y que eran: Mes del año, diagnósticos de tipo de lesión, tipo y número de dientes afectados, género, edad, lugar de ocurrencia del accidente y causa, determinándose la frecuencia de cada una de estas variables.

La muestra en estudio la conformaron 604 niños y niñas, la que luego se desagregó para el análisis de los datos en dentición temporal, en el rango etario de 0 años (incisivos temporales se encuentran habitualmente erupcionados antes del primer año de vida) a 10 años; mientras que la dentición definitiva se presentaba en un rango de edad entre los 5 años (edad en que pueden haber erupcionado los incivos definitivos mandibulares) y 14 años 11 meses. Rango etario considerado para la población infantil, y que recibe atención en la Clínica de Odontopediatría

Por otro lado, considerando que había niños con dentición mixta y más de un diente afectado, hubo pacientes con ambas denticiones involucradas. Los datos se tabularon en el programa Excel versión Office 2007 y se determinó la frecuencia con el programa Systat v.13.

Cabe consignar que las necesidades de tratamiento de todos los niños fueron canalizadas en el CASR.

\section{RESULTADOS}

Entre los meses de Enero y Diciembre del año 2010 se examinaron 604 pacientes niños, derivados a la Clínica de Odontopediatría del Complejo Asistencial Dr. Sotero del Río, con diagnostico de TDA, siendo afectados 636 dientes definitivos y 414 dientes temporales. Para ambas denticiones en conjunto $58.77 \%(n=355)$ de los niños tuvieron afectados más de un diente, y el detalle se describe a continuación, un solo diente comprometido $(\mathrm{N}=249)$, dos dientes $(\mathrm{N}=266), 3$ dientes $(\mathrm{N}=87)$ y 4 dientes $(\mathrm{N}=2)$.

Para facilitar la comprensión del estudio se analizó la dentición definitiva primero y posteriormente la dentición temporal.

\section{Resultados en Dentición Definitiva}

En dentición definitiva los meses del año en que se observó mayor frecuencia de TDA fueron Mayo con $15.36 \%(n=59)$, Abril $13.02 \%$ $(n=50)$ y Noviembre $13.02 \%(n=50)$ (Tabla 1$)$.

Con respecto a los diagnósticos el detalle de la distribución es el siguiente: fracturas coronarias no complicadas $(40.88 \%, n=260)$ subluxación $(22.17 \%, n=141)$ y concusión $(19.5 \%, n=124)$, los demás diagnósticos se presentaron en menores porcentajes como fracturas coronarias complicadas $(5.97 \%, \mathrm{n}=38)$, luxaciones laterales $(4.87 \%$, $\mathrm{n}=31)$, avulsiones $(2.37 \%, \mathrm{n}=15)$, luxación extrusiva $(1.57 \%, \mathrm{n}=10)$, fractura radicular $(1.26 \%, \mathrm{n}=8)$ y luxación intrusiva $(0.31 \%, \mathrm{n}=2)$ (Tabla 2$)$.

\section{Distribución de Lesiones Traumáticas de Acuerdo a los Dientes Afectados}

Los dientes maxilares afectados constituyeron un 88,99\% $(n=566)$ de los casos, los incisivos centrales tuvieron la mayor frecuencia de traumatismos alcanzando un $77.66 \%(n=494)$, luego los incisivos laterales un $10.22 \%(n=65)$ y los resultados de la suma de los otros dientes maxilares afectadas es $1.1 \%(n=7)$ (Tabla 3$)$.

Los dientes mandibulares afectados constituyeron un $11.01 \%$ $(n=70)$ del total de dientes definitivos afectados y al igual que en el maxilar las lesiones se distribuyen principalmente en los incisivos centrales, con un $7.39 \%(n=47)$, luego los incisivos laterales con $2.2 \%(n=14)$, los otros dientes mandibulares comprometidos correspondieron al $1.43 \%(n=9)$ y se pueden observar en la Tabla 3.

\section{Distribución por Género y Edad}

En relación a la distribución por género un $64.97 \%(n=269)$ correspondió a niños y un $35.03 \%(n=145)$ correspondió a niñas (Tabla 4). Las edades se distribuyeron entre los 5 años y 14 años 11 meses y en ambos géneros las edades más frecuentes fueron entre los 7 y 9 años con un $52.72 \%$ en niños y un $58.6 \%$ en niñas, más detalle en Tabla 4.

\section{Causas de Lesiones}

El $76 \%$ de los casos observados correspondió a accidentes escolares $(n=292)$ (Tabla 5) y las principales causas fueron caídas $68.49 \%(n=263)$ y de estas $68.44 \%(n=180)$ correspondieron a caídas en el colegio. La frecuencia de las otras causas fueron golpe con objeto $10.16 \%(n=39)$, choque contra pared, puerta o compañero $10.42 \%$ $(n=40)$, golpe con puño, pie o parte de cuerpo $9.64 \%(n=37)$ y atropello $1.3 \%(n=5)($ Tabla 6$)$.

\section{Resultados en Dentición Temporal}

En dentición temporal los meses del año en que se observó mayor frecuencia de TDA fueron Noviembre con $15.86(n=36)$, Febrero $14.98 \%(n=34)$ y Abril $12.78 \%(n=29)$ (Tabla 1$)$.

El detalle de la distribución de los diagnósticos es el siguiente: subluxación $(39.61 \%, \mathrm{n}=164)$, luxaciones laterales $(20.77 \%$, $n=86)$, concusión $(11.84 \%, n=49)$, avulsiones $(7.25 \%, n=30)$, fracturas coronarias no complicadas $(6.76 \%, n=28)$, luxación intrusiva $(4.83 \%$ $n=20)$, luxación extrusiva $(3.62 \%, n=15)$, fractura radicular $(2.66 \%, n=11)$ y fracturas coronarias complicadas $(1.69 \%, n=7)$ (Tabla 2$)$.

\section{Distribución de Lesiones Traumáticas de Acuerdo a los Dientes Afectados}

Los dientes maxilares afectados constituyeron un $96.38 \%$ $(n=399)$ de los casos. Los incisivos centrales superiores se presentaban en un $82.13 \%(n=340)$ y los incisivos laterales en un $12.56 \%(n=52)$, los resultados de los otros dientes los podemos observar en la Tabla 3.

Los dientes mandibulares afectados constituyeron un $3.62 \%$ $(n=15)$ del total y al igual que en el maxilar las lesiones se distribuyen principalmente en los incisivos centrales un $2.41 \%(n=10)$, incisivos laterales $0.97(n=4)$ y el segundo molar temporal $0.24 \%(n=1)$.

\section{Distribución por Género y Edad}

En relación a la distribución por género un $62.56 \%(n=142)$ correspondió a niños y un $37.44 \%(n=85)$ correspondió a niñas (Tabla 4). Las edades se distribuyeron entre los 0 años y 10 años, la frecuencia 
tanto en niños como en niñas fue al año de $15.42 \%(n=35)$, a los 2 años de $20.26 \%(n=46)$, a los 3 años de $18.06 \%(n=41)$, a los 4 años $17.62 \%$ $(n=40)$, a los 5 años $12.33 \%(n=28)$, a los 6 años $12.33 \%(n=28)$ y más detalle en Tabla 4

\section{Causas de Lesiones}

El $31.72 \%$ de los casos observados correspondió a accidentes escolares $(n=72)$ (Tabla 5) y las principales causas fueron caídas $81.5 \%$ $(n=185)$ y de estas $35.68 \%(n=66)$ ocurrieron en la casa, de las otras causas golpe con objeto $11.01 \%(n=25)$, golpe con puño, pie o parte de cuerpo $3.96 \%(n=9)$, choque contra pared o puerta $2.64 \%(n=6)$ y atropello $0.88 \%(n=2)$ (Tabla 6$)$.

Tabla 1. Distribución y frecuencia de dientes temporales y definitivos según el mes del año.

\begin{tabular}{|l|cc|cc|}
\hline & \multicolumn{2}{|c|}{ DENTICION TEMPORAL } & \multicolumn{2}{c|}{ DENTICION DEFINITIVA } \\
\hline Meses & $\mathrm{n}$ & $(\%)$ & $\mathrm{n}$ & $(\%)$ \\
\hline Enero & 4 & 1.76 & 4 & 1.04 \\
\hline Febrero & 34 & 14.98 & 9 & 2.34 \\
\hline Marzo & 16 & 7.05 & 24 & 6.25 \\
\hline Abril & 29 & 12.78 & 50 & 13.02 \\
\hline Mayo & 22 & 9.7 & 59 & 15.36 \\
\hline Junio & 15 & 6.61 & 37 & 9.64 \\
\hline Julio & 8 & 3.5 & 13 & 3.39 \\
\hline Agosto & 15 & 6.61 & 47 & 12.24 \\
\hline Septiembre & 9 & 3.97 & 37 & 9.64 \\
\hline Octubre & 19 & 8.37 & 30 & 7.81 \\
\hline Noviembre & 36 & 15.86 & 50 & 13.02 \\
\hline Diciembre & 20 & 8.81 & 24 & 6.25 \\
\hline Total & $\mathbf{2 2 7}$ & $\mathbf{1 0 0}$ & $\mathbf{3 8 4}$ & $\mathbf{1 0 0}$ \\
\hline
\end{tabular}

Tabla 2. Distribución y frecuencia de diagnósticos en dentición temporal y definitiva.

\begin{tabular}{|c|l|cc|cc|}
\hline \multirow{4}{*}{$\begin{array}{c}\text { Tejidos } \\
\text { Duros }\end{array}$} & & \multicolumn{2}{|c|}{ DENTCIÓNTEMPORAL } & \multicolumn{2}{|c|}{ DENTCIÓNDEFNNTINA } \\
\cline { 2 - 6 } & Diagnósticos & $\mathbf{n}$ & $(\%)$ & $\mathbf{n}$ & $(\%)$ \\
\cline { 2 - 6 } & FCNC & 28 & 6.76 & 260 & 40.88 \\
\cline { 2 - 6 } & FCC & 7 & 1.69 & 38 & 5.97 \\
\cline { 2 - 6 } & FR & 11 & 2.66 & 8 & 1.26 \\
\cline { 2 - 6 } & FA & 4 & 0.97 & 7 & 1.1 \\
\hline \multirow{4}{*}{$\begin{array}{c}\text { Tejidos } \\
\text { de Soporte }\end{array}$} & CON & 49 & 11.84 & 124 & 19.50 \\
\cline { 2 - 6 } & SUBL & 164 & 39.61 & 141 & 22.17 \\
\cline { 2 - 6 } & LE & 15 & 3.62 & 10 & 1.57 \\
\cline { 2 - 6 } & LL & 86 & 20.77 & 31 & 4.87 \\
\cline { 2 - 6 } & LI & 20 & 4.83 & 2 & 0.31 \\
\cline { 2 - 6 } & $\mathrm{A}$ & 30 & 7.25 & 15 & 2.37 \\
\hline & Total & 414 & 100 & $\mathbf{6 3 6}$ & 100 \\
\hline
\end{tabular}

FCNC: Fractura coronaria no complicada. FCC: Fractura coronaria complicada. FR: Fractura radicular FA: Fractura alveolar. CON: Concusión. SUBL: Subluxación. LE: Luxación extrusiva. LL: Luxación lateral. Ll: Luxación intrusiva. A: Avulsión.

Tabla 3. Frecuencia y distribución de dientes temporales y definitivos de la muestra.

\begin{tabular}{|c|c|c|c|c|c|}
\hline \multirow{7}{*}{$\begin{array}{l}\text { Maxilar } \\
\text { Superior }\end{array}$} & & \multicolumn{2}{|c|}{ TEMPORALES } & \multicolumn{2}{|c|}{ DEFINITIVOS } \\
\hline & Tipo de Dientes & $\mathrm{n}$ & $(\%)$ & $\mathrm{n}$ & (\%) \\
\hline & Incisivos centrales & 340 & 82.13 & 494 & 77.66 \\
\hline & Incisivos laterales & 52 & 12.56 & 65 & 10.22 \\
\hline & Caninos & 4 & 0.97 & 6 & 0.94 \\
\hline & $1^{\circ} \mathrm{molar}$ temporal $/ 1^{\circ} \mathrm{premolar}$ & 2 & 0.48 & & \\
\hline & $2^{\circ} \mathrm{molar}$ temporal/ $/ 2^{\circ}$ premolar & 1 & 0.24 & 1 & 0.16 \\
\hline \multirow{6}{*}{$\begin{array}{l}\text { Maxilar } \\
\text { Inferior }\end{array}$} & Incicivos centrales & 10 & 2.41 & 47 & 7.39 \\
\hline & Incisivos laterales & 4 & 0.97 & 14 & 2.20 \\
\hline & Caninos & & & 4 & 0.63 \\
\hline & $1^{\circ} \mathrm{molar}$ temporal $/ 1^{\circ} \mathrm{premolar}$ & & & 2 & 0.32 \\
\hline & $2^{\circ} \mathrm{molar}$ temporal $/ 2^{\circ}$ premolar & 1 & 0.24 & 1 & 0.16 \\
\hline & $1^{\circ} \mathrm{molar}$ definitivo & & & 2 & 0.32 \\
\hline & Total & 414 & 100 & 636 & 100 \\
\hline
\end{tabular}

Tabla 4. Distribución y frecuencia por género y edad en dentición temporal y definitiva.

\begin{tabular}{|l|cc|cc|cc|cc|}
\hline & \multicolumn{3}{|c|}{ MASCULINO } & \multicolumn{4}{c|}{ FEMENINO } \\
\hline & \multicolumn{2}{|c|}{ Temporal } & \multicolumn{2}{|c|}{ Definitiva } & \multicolumn{2}{|c|}{ Temporal } & \multicolumn{2}{|c|}{ Definitiva } \\
\hline Edad & $\mathbf{n}$ & $\mathbf{( \% )}$ & $\mathbf{n}$ & $\mathbf{( \% )}$ & $\mathbf{n}$ & $(\%)$ & $\mathbf{n}$ & $(\%)$ \\
\hline 0 & 1 & 0.70 & & & 1 & 1.18 & & \\
\hline 1 & 21 & 14.79 & & & 14 & 16.47 & & \\
\hline 2 & 28 & 19.72 & & & 18 & 21.18 & & \\
\hline 3 & 23 & 16.20 & & & 18 & 21.18 & & \\
\hline 4 & 26 & 18.31 & & & 14 & 16.47 & & \\
\hline 5 & 21 & 14.79 & & & 7 & 8.24 & 3 & 2.07 \\
\hline 6 & 17 & 11.97 & 8 & 3.35 & 11 & 12.94 & 7 & 4.83 \\
\hline 7 & 3 & 2.11 & 38 & 15.90 & 1 & 1.18 & 29 & 20.00 \\
\hline 8 & 1 & 0.70 & 47 & 19.67 & 1 & 1.18 & 32 & 22.07 \\
\hline 9 & & & 41 & 17.15 & & & 24 & 16.55 \\
\hline 10 & 1 & 0.70 & 27 & 11.30 & & & 16 & 11.03 \\
\hline 11 & & & 28 & 11.72 & & & 12 & 8.28 \\
\hline 12 & & & 18 & 7.53 & & & 11 & 7.59 \\
\hline 13 & & & 18 & 7.53 & & & 10 & 6.90 \\
\hline 14 & & & 14 & 5.86 & & & 1 & 0.69 \\
\hline Total & 142 & 100 & 239 & 100 & 85 & 100 & 145 & 100 \\
\hline
\end{tabular}

Tabla 5. Distribución y frecuencia según el tipo de accidente, género y dentición.

\begin{tabular}{|l|cc|cc|cc|cc|}
\hline \multirow{2}{*}{} & \multicolumn{2}{|c|}{ ACCIDENTE } & \multicolumn{2}{c|}{ ESCOLAR } & \multicolumn{2}{c|}{ ACCIDENTE } & \multicolumn{2}{c|}{ NO ESCOLAR } \\
\cline { 2 - 10 } & \multicolumn{2}{|c|}{ Temporal } & \multicolumn{2}{c|}{ Definitiva } & \multicolumn{2}{|c|}{ Temporal } & \multicolumn{2}{c|}{ Definitiva } \\
\cline { 2 - 10 } & $\mathbf{n}$ & $(\%)$ & $\mathbf{n}$ & $(\%)$ & $\mathbf{n}$ & $(\%)$ & $\mathbf{n}$ & $(\%)$ \\
\hline Masculino & 47 & 65.28 & 185 & 63.36 & 95 & 61.29 & 54 & 61.29 \\
\hline Femenino & 25 & 34.72 & 107 & 36.64 & 60 & 38.71 & 38 & 38.71 \\
\hline Total & 72 & 100 & 292 & 100 & 155 & 100 & 92 & 100 \\
\hline
\end{tabular}

Tabla 6. Distribución y frecuencia de causas de traumatismos en dentición temporal y definitiva.

\begin{tabular}{|l|cc|cc|cc|cc|}
\hline \multirow{2}{*}{} & \multicolumn{3}{|c|}{ MASCULINO } & \multicolumn{3}{c|}{ FEMENINO } \\
\cline { 2 - 11 } & \multicolumn{2}{|c|}{ Temporal } & \multicolumn{2}{|c|}{ Definitiva } & \multicolumn{2}{c|}{ Temporal } & \multicolumn{2}{c|}{ Definitiva } \\
\hline Causa & $\mathbf{n}$ & $\mathbf{( \% )}$ & $\mathbf{n}$ & $\mathbf{( \% )}$ & $\mathbf{n}$ & (\%) & $\mathbf{n}$ & (\%) \\
\hline $\begin{array}{l}\text { Caída de (bicicleta, } \\
\text { triciclo o andador) }\end{array}$ & 11 & 7.75 & 9 & 3.77 & 3 & 3.53 & 6 & 4.14 \\
\hline Caída en la casa & 30 & 21.13 & 14 & 5.86 & 36 & 42.35 & 11 & 7.59 \\
\hline Caída de coche & 1 & 0.70 & 0 & & 0 & & 0 & \\
\hline Caída de moto & 2 & 1.41 & 2 & 0.84 & 0 & & 0 & \\
\hline Caída de escalera & 10 & 7.04 & 3 & 1.26 & 1 & 1.18 & 3 & 2.07 \\
\hline Caída de la cama & 10 & 7.04 & 0 & & 5 & 5.88 & 1 & 0.69 \\
\hline Caída en piscina & 0 & & 0 & & 2 & 2.35 & 2 & 1.38 \\
\hline Caída de silla & 0 & & 1 & 0.42 & 0 & & 0 & \\
\hline Caída en la calle & 15 & 10.56 & 18 & 7.53 & 8 & 9.41 & 13 & 8.97 \\
\hline $\begin{array}{l}\text { Caída en el } \\
\text { colegio }\end{array}$ & 35 & 24.65 & 115 & 48.12 & 16 & 18.88 & 65 & 44.83 \\
\hline $\begin{array}{l}\text { Choque con pared } \\
\text { puerta u otro }\end{array}$ & 3 & 2.11 & 13 & 5.44 & 3 & 3.53 & 8 & 5.52 \\
\hline $\begin{array}{l}\text { Choque con } \\
\text { compañero }\end{array}$ & 0 & & 11 & 4.60 & 0 & & 8 & 5.52 \\
\hline atropello & 2 & 1.41 & 2 & 0.84 & 0 & & 3 & 2.07 \\
\hline Golpe con objeto & 16 & 11.27 & 25 & 10.46 & 9 & 10.59 & 14 & 9.66 \\
\hline $\begin{array}{l}\text { Golpe con puño u } \\
\text { otra parte del cuerpo }\end{array}$ & 7 & 4.93 & 26 & 10.88 & 2 & 2.35 & 11 & 7.59 \\
\hline Total & 142 & 100 & 239 & 100 & 85 & 100 & 145 & 100 \\
\hline
\end{tabular}

\section{DISCUSIÓN}

Dentro de las atenciones de urgencia odontológicas figuran los TDA como una de las causas de consulta más frecuente, la Guía Clínica de Urgencia Odontológica Ambulatoria, Minsal 2007, menciona que estudios en niños realizados en Chile en el Hospital de Niños Roberto del Río, señalan que en el año 2000, las lesiones traumáticas 
dentoalveolares representaron el $16.8 \%$ de las atenciones de urgencia en niños, con un $44.4 \%$ para la dentición permanente y un $55.6 \%$ en la dentición temporal(11). En el Servicio de Urgencia del CASR 6.6\% de las atenciones odontológicas son por TDA y como en nuestro país existen pocos estudios en niños, salvo los realizados por Onetto 1994 en Valparaíso, Soto 2007 a nivel nacional y Díaz 2010 en Temuco, es de importancia tener mayor información al respecto.

EI SMSO es uno de los Servicios de Salud con más demanda asistencial en la Región Metropolitana y $23.4 \%$ de la población infantil recibe atención aquí, siendo la Clínica de Odontopediatría del CASR el único referente de atención odontopediátrica de todo el SSMSO, por lo cual la información obtenida del presente estudio tiene valides en sí misma; además "la información adicional tiene un valor real, en cuanto resuelve la incertidumbre y aumenta las ganancias esperadas de salud" (Espinoza et al., 2010).

Analizando los resultados obtenidos podemos señalar que, en relación a las variaciones estacionales, en la dentición definitiva figuró el mes de Mayo con mayor número de TDA, otoño en nuestro país, seguido de los meses de Abril y Noviembre, época en que los niños están en actividades escolares, lo que concuerda con el alto porcentaje de accidentes escolares observados en el presente estudio (76\%), parecido al estudio de Díaz $2010^{(6)}$, que obtuvo un porcentaje de $57.1 \%$; a su vez Andreasen menciona que la frecuencia de estas lesiones aumenta en invierno ${ }^{(17)}$. Glendor 2008 menciona que es necesaria investigación para dilucidar la relación existente entre los factores ambientales y de comportamiento con los TDA ${ }^{(1)}$.

Mientras que en dentición temporal se observó mayor frecuencia de TDA en primavera-verano (53.74\%), época del año en que los niños pequeños realizan más actividades al aire libre.

De acuerdo al tipo de tejido afectado, en la dentición definitiva se mantuvo la tendencia internacional, y las fracturas coronarias no complicadas fueron las más frecuentes ${ }^{(5,6,18-21)}$, a su vez las avulsiones presentaron un porcentaje de $2.37 \%$, similar a lo observado por otros autores que concuerdan que es una de las lesiones más serias y el pronóstico es dependiente de las acciones que se realicen en el lugar del accidente, inmediatamente ocurrida la avulsión ${ }^{(4)}$, lo cual ratifica que se deben realizar campañas de información enfocadas a la acción oportuna frente a la avulsión de un diente definitivo. Mientras que en dentición temporal en nuestro estudio la mayor frecuencia de los diagnósticos fue de subluxaciones $(39.61 \%$ ) y los estudios internacionales mencionan tanto subluxaciones como luxaciones ${ }^{(5,6,17,20,22,23)}$.
En el presente estudio se mantuvo la tendencia internacional siendo los incisivos centrales maxilares los dientes más afectados tanto en dentición definitiva como temporal ${ }^{(1,17,18,20)}$, y el $58.77 \%$ de todos los niños tuvo más de un diente afectado, lo que nos parece interesante ya que se pueden lesionar ambas denticiones al mismo tiempo y por tanto los tratamientos son diversos y personales, de acuerdo a las condiciones de cada niño. El resultado no seria comparable al estudio nacional de Soto L, 2007, porque este último se realizo solo en niños de 12 años.

En dentición definitiva se observó mayor frecuencia en niños (género masculino) al igual como lo menciona la literatura ${ }^{(1,6,10,17,19,24)}$. Pero cabe destacar como lo menciona Traebert et al. que las niñas están cada vez más expuestas a los mismos factores de riesgo que los niños, lo cual es característico de las sociedades occidentales modernas ${ }^{(25)}$.

En dentición temporal fue el género masculino el más frecuente y en la literatura existen diferentes opiniones al respecto(17,20,23,26).

En dentición temporal y definitiva las caídas siguen siendo la principal causa al igual como lo menciona la bibliografía ${ }^{(2,5,17,19,21,23,27,28,29)}$, y dentro de estas, las caídas en los colegios fueron las más frecuentes $68.44 \%$. Aunque la literatura es discrepante ${ }^{(21)}$, cabe mencionar que actualmente las causas han ido modificándose por las nuevas costumbres y formas de relacionarse entre los niños ${ }^{(2)}$.

En dentición temporal las caídas ocurridas en el hogar fueron de $35.68 \%$ lo que concuerda con estudios de Glendor $2009^{(2)}$, y un porcentaje no despreciable de $31.7 \%$, de los dientes temporales fueron traumatizados en establecimientos educacionales, lo que implica que las medidas de prevención deben ser implementadas o aumentadas tanto en los colegios como en establecimientos educacionales prescolares, ya que es cada vez mayor el número de niños que asisten a jardines infantiles y salas cuna.

\section{CONFLICTOS DE INTERÉS}

Los autores declaran no tener conflictos de interés.

\section{AGRADECIMIENTOS}

Al Dr. Benjamin Martinez Rondanelli por su valiosa colaboración.

\section{REFERENCIAS BIBLIOGRÁFICAS}

1. Glendor U. Epidemiology of traumatic dental injuries - a 12 year review of the literature. Dent Traumatol, 2008; 24: 603-611.

2. Glendor U. Aetiology and risk factors related to traumatic dental injuries - a review of the literature. Dent Traumatol, 2009; 25: 19-31.

3. DiAngelis AJ, Andreasen JO, Ebeleseder KA, Kenny DJ, Trope M, Sigurdsson A, Andersson L, Bourguignon C, Flores MT, Hicks ML, Lenzi AR, Malmgren B, Moule AJ, Pohl Y, Tsukiboshi M. International Association of Dental Traumatology guidelines for the managementof traumatic dental injuries: 1. Fractures and luxations of permanent teeth. Dent Traumatol, 2012; 28: 2-12.

4. Andersson L, Andreasen JO, Day P, Heithersay G, Trope M, DiAngelis AJ, Kenny DJ, Sigurdsson A, Bourguignon C, Flores MT, Hicks ML, Lenzi AR, Malmgren B. Moule AJ, Tsukiboshi M. International Association of Dental Traumatology guidelines for the managementof traumatic dental injuries: 2. Avulsion of permanent teeth. Dent Traumatol, 2012; 28: 88-96.

5. Onetto JE, Flores MT, Garbarino ML. Dental trauma in children and adolescents in Valparaiso. Chile. Endod Dent Traumatol, 1994; 10: 223-227.

6. Díaz JA, Bustos L, Brandt AC, Fernández BE. Dental injuries among children and adolescents aged 1-15 years attending to public hospital in Temuco, Chile. Dent Traumatol, 2010; 26: 254-226.

7. Fodor A, Navarrete E, Caceres E, Munoz F. Atención de urgencia odontológica en el Hospital Urgencia Asistencia Pública. Rev Dent Chile, 2005; 96(3): 3-6.

8. Gantz A, Figueroa L, Guzman CL, Rudolph M. Relacion entre traumatismos buco-dentarios y anomalias dentomaxilo faciales. Rev Dent Chile, 2003; 94(3): 3-6. 9. Acevedo JP, Cooper H, Tirreau V, Núñez F. Descripción del traumatismo dentoalveolar en pacientes adultos. Rev Dent Chile, 2006; 97(1): 8-13.

10. Soto L, Tapia R, Jara G y col. Diagnóstico Nacional de Salud Bucal del adolescente de 12 años y evaluación del grado de cumplimiento de los Objetivos Sanitarios de Salud Bucal 2000-2010. Facultad de Odontología Universidad Mayor. Chile 2007. 11. Ministerio de Salud. Guía Clínica Urgencia Odontológica Ambulatoria. Santiago, Chile: Minsal, 2007.

12. Ministerio de Salud. Diagnóstico epidemiológico Servicio de Salud Sur Oriente, Departamento Control de Gestión, Santiago, Chile: MINSAL, 2010.

13. Ministerio de Salud. Estadísticas/DEIS, MINSAL, Chile, 2010.

14. Ministerio de Salud. Metas 2011-2020 Estrategia Nacional de Salud para el cumplimiento de los Objetivos Sanitarios de la Década 2011-2020. MINSAL, Gobierno de Chile.

15. Lewis C, Lynch H, Johnston B. Dental complaints in emergency departments: A national perspective. Ann Emerg Med, 2003 Jul; 42(1): 93-99.

16. Andreasen JO, Andreasen FM, Andersson L, editors. Textbook and color atlas of traumatic injuries to the teeth, $4^{\text {th }}$ edn. Oxford, UK: Wiley-Blackwell; 2007. p. 444-488. 17. Andreasen JO, Backland LK, Flores MT, Andreasen FM, Andreasen L. Traumatic dental injuries: A manual, $3^{\text {rd }}$ Edition, 2011.

18. Piovesan C, Abella C, Ardenghi TM. Child oral health-related quality of life and socioeconomic factors associated with traumatic dental injuries in schoolchildren. Oral Health Prev Dent, 2011; 9(4): 405-411.

19. Kumar A, Bansal V, Veeresha KL, Sogi GM. Prevalence of traumatic dental injuries among 12- to 15-year-old schoolchildren in Ambala district, Haryana, India. Oral Health Prev Dent, 2011; 9(3): 301-305.

20. Coutinho TC, Cajazeira MR. Retrospective study on the occurrence of primary incisor trauma in preschool children of a low-income area in Brazil. Eur J Paediatr Dent, 2011 Sep; 12(3): 159-162.

21. Ministerio de Salud. Guía Clínica AUGE, Urgencias Odontológicas Ambulatorias Santiago, Chile: MINSAL, 2011.

22. Flores MT. Traumatic injuries in the primary dentition. Dent Traumatol, 2002 18: $287-298$

23. de Amorim L de F, da Costa LR, Estrela C. Retrospective study of traumatic dental injuries in primary teeth in a Brazilian specialized pediatric practice. Dent Traumatol, 2011 Oct; 27(5): 368-373.

24. Ankola AV, Hebbal M, Sharma R, Nayak SS. Traumatic dental injuries in primary school children of South India - a report from district-wide oral health survey. Dent Traumatol, 2012 Apr 2. doi: 10.1111/j.1600-9657.2012.01139.x.

25. Traebert J, Bittencourt DD, Peres KG, Peres MA, de Lacerda JT, Marcenes W. A etiology and rates of treatment of traumatic dental injuries among 12-year-old school children in a town in southern Brazil. Dent Traumatol, 2006; 22: 173178.

26. Tümen EC, Adigüzel O, Kaya S, Uysal E, Yavuz I, Ozdemir E, Atakul F. Incisor trauma in a Turkish preschool population: Prevalence and socio-economic risk factors. Community Dent Health, 2011 Dec; 28(4): 308-312.

27. Fried I, Erickson P, Schwartz S, Keenan K. Subluxation injuries of maxillary primary anterior teeth: Epidemiologyand prognosis of 207 traumatized teeth. Pediatr Dent, 1996; 18: 145-151.

28. Perez R, Berkowitz R, Mcllveen L, Forrester D. Dental trauma in children: A survey. Endod Dent Traumatol, 1991; 7: 212-213.

29. Soporowski NJ, Allred EN, Needleman HL. Luxation injuriesof primary anterior teeth - prognosis and related correlates. Pediatric Dent, 1994; 16: 96-101. 